\section{Évolution et adaptation fonctionnelle des arbres tropicaux : le cas du genre Guibourtia Benn.}

\section{Félicien Tosso}

\section{RÉSUMÉ}

La présente thèse s'intéresse aux mécanismes à l'origine de la diversification des espèces d'arbres tropicaux. Elle utilise le genre Guibourtia Benn. (Fabaceae-Detarioideae) comme modèle biologique afin de comprendre les mécanismes historiques, biologiques et environnementaux à l'origine de la diversité de ce genre. Plus particulièrement, elle vise, d'une part, à étudier, au niveau interspécifique, le rôle relatif des forces évolutives neutres et de sélection dans la diversification du genre Guibourtia et, d'autre part, au niveau intraspécifique, à questionner les causes de la différenciation des populations de trois espèces de Guibourtia. Au niveau interspécifique, la phylogénie datée basée sur le séquençage du génome chloroplastique complet a globalement confirmé la taxonomie actuelle. Elle a montré une diversification au milieu du Miocène en trois clades qui sont décrits aujourd'hui comme des sous-genres (Guibourtia, Gorskia et Pseudocopaiva). Elle démontre en outre que deux espèces américaines sont issues d'une migration de l'Afrique vers l'Amérique à la fin du Miocène. Il est également apparu que certains traits morphologiques ont été sélectionnés de manière convergente au sein des différents clades du genre Guibourtia en fonction des niches climatiques des espèces. Ce dernier résultat a été consolidé au moyen d'une expérimentation écophysiologique prouvant que la lumière constitue un important facteur de sélection et de différenciation adaptative entre certaines espèces du genre. Au niveau intraspécifique, une étude de phylogéographie a permis de mettre en évidence que les barrières biogéographiques chez $G$. ehie et les gradients climatiques chez $G$. coleosperma auraient contribué à la différenciation génétique des populations. En outre, cette étude montre une forte différenciation entre les populations de G. ehie d'Afrique de l'Ouest et d'Afrique centrale, en lien avec quelques traits morphologiques, ce qui préjuge de l'existence d'une nouvelle espèce. Enfin, chez G. tessmannii, espèce aux fruits déhiscents et graines arillées, nous avons identifié les principaux disperseurs dont les calaos (Ceratogymna atrata) qui pourraient contribuer à une dispersion à longue distance, influençant la structure spatiale de la variation génétique des populations. Cette thèse, en utilisant le genre Guibourtia comme modèle d'étude, a apporté de nouveaux éléments à la compréhension des mécanismes qui génèrent la diversité au sein des espèces d'arbres.

Mots-clés : évolution, adaptation, arbres tropicaux, Fabaceae-Detarioideae, Afrique.

\section{Evolution and functional adaptation of tropical tree species: a case study on Guibourtia Benn.}

\section{ABSTRACT}

This thesis investigates diversification processes in tropical tree species, using the genus Guibourtia Benn. as a biological model (Leguminosae-Detarioideae). The aim is to understand the historical, biological and environmental mechanisms that account for the diversity of the genus. In particular, this PhD aims to: (1) understand the relative role, at the interspecies level, of neutral and selection forces in the diversification of Guibourtia species, by combining molecular phylogeny with the characterization of functional traits and ecological niches; and (2) at the intraspecies level, investigate the causes of population differentiation in three Guibourtia species. At the interspecies level, the molecular phylogeny dating based on whole plastid genome sequencing was congruent with the current taxonomy (the species were generally monophyletic). The dating showed diversification in the Middle Miocene into three clades corresponding to the subgenera Guibourtia, Gorskia and Pseudocopaiva. It also showed that two neo-Tropical species resulted from migration from Africa to South America during the Late Miocene. In considering climate niches, it was also demonstrated that the selection of some morphological traits was convergent within the different clades. This result was confirmed by an ecophysiological experiment, which showed that light was an important factor of selection and adaptive differentiation between three species (G. ehie, G. coleosperma and $G$. tessmannii). At the intraspecies level, a phylogeographic study of two Guibourtia species revealed that biogeographic barriers climatic gradients appear to have contributed to the genetic differentiation of G. ehie and G. coleosperma populations respectively. This study also investigates the limits of $G$. ehie distribution, since its populations showed a strong genetic differentiation between West and Central Africa, associated with diagnostic morphological features. Finally, in G. tessmannii, a species with dehiscent fruits and arillate seeds, we identified the main dispersal species, including hornbills (Ceratogymna atrata), that may have contributed to long-distance gene flows influencing the spatial structure of genetic variation among populations. This thesis, using the Guibourtia genus as a biological model, has brought new material to help understand the mechanisms generating diversity in tropical tree species.

Keywords: evolution, adaptation, tropical trees, Fabaceae-Detarioideae, Africa

\section{Evolución y adaptación funcional de los árboles tropicales: el caso del género Guibourtia Benn.}

\section{RESUMEN}

La presente tesis se interesa por los mecanismos causantes de la diversificación de especies de árboles tropicales. Utiliza el género Guibourtia Benn. (Fabaceae-Detarioideae) como modelo biológico para comprender los mecanismos históricos, biológicos y medioambientales, que originan la diversidad de este género. De forma más particular, el estudio pretende: (1) estudiar, a nivel interespecífico, el rol relativo de las fuerzas evolutivas neutras y de selección en la diversificación del género Guibourtia, y (2), a nivel intraespecífico, cuestionar las causas de la diferenciación de las poblaciones de tres especies de Guibourtia. Al nivel interespecífico, la filogenia fechada basada en la secuenciación del genoma cloroplástico completo ha confirmado globalmente la taxonomía actual. Ha mostrado una diversificación en el medio del Mioceno en tres clados que se describen actualmente como subgéneros (Guibourtia, Gorskia y Pseudocopaiva). Demuestra además que dos especies americanas provienen de la migración de África hacia América al final del Mioceno. Se ve asimismo que ciertos rasgos morfológicos fueron seleccionados de forma convergente en el seno de los diferentes clados del género Guibourtia en función de los nichos climáticos de las especies. Este último resultado fue consolidado mediante una experimentación ecofisiológica que prueba que la luz constituye un importante factor de selección y de diferenciación adaptativa entre ciertas especies del género. A nivel intraespecífico, un estudio filogeográfico permitió poner en evidencia que las barreras biogeográficas en $G$. ehie y los gradientes climáticos en $G$. coleosperma habrían contribuido a la diferenciación genética de las poblaciones. Además, este estudio demuestra una fuerte diferenciación entre las poblaciones de G. ehie de África occidental y de África central en relación con algunos rasgos morfológicos, lo que anticipa la existencia de una nueva especie. Finalmente, para $G$. tessmannii, especie con frutos dehiscentes y semillas ariladas, hemos identificado a los principales dispersores, entre ellos los cálaos (Ceratogymna atrata), que podrían contribuir a una dispersión a larga distancia e influyen en la distribución espacial y la variación genética de las poblaciones. Esta tesis, que utiliza el género Guibourtia como modelo de estudio, ha aportado nuevos elementos a la comprensión de los mecanismos que generan la diversidad en el seno de las especies arbóreas.

Palabras clave: evolución, adaptación, árboles tropicales, Fabaceae-Detarioideae, África.
Doi : $10.19182 / \mathrm{bft} 2018.337 . a 31632$

Droit d'auteur (c) 2018, Bois et Forêts des Tropiques @ C Cirad

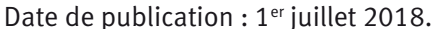


Bois et Forêts des Tropiques - ISSN: L-0006-57

Volume $337-3^{\text {rd }}$ quarter - July 2018 - p. $79-80$

Phylogénie du genre Guibourtia montrant la structuration en trois sous-genres (Guibourtia en jaune, Gorskia en vert et Pseudocopaiva en rouge) comme décrit par Léonard (1949).

Un encadré rouge sur la phylogénie montre les deux espèces américaines qui seraient

issues d'une migration de l'Afrique vers l'Amérique à la fin du Miocène $(-11,78 \mathrm{Ma})$.

Figure 1.

Phylogeny of the genus Guibourtia showing the structure in three subgenera (Guibourtia in yellow, Gorskia in green and Pseudocopaiva in red) as described by Léonard (1949). The red squares on the phylogeny shows the two neo-Tropical species that appear to have migrated from Africa to South America at the end of the Miocene ( 11.78 Ma).

\section{Figura 1.}

Filogenia del género Guibourtia que muestra la estructuración en tres subgéneros (Guibourtia en amarillo, Gorskia en verde y Pseudocopaiva en rojo) como describe Léonard (1949). Un cuadro rojo sobre la filogenia muestra las dos especies americanas que provendrían de una migración de África hacia América al final del Mioceno $(\sim 11,78 \mathrm{Ma})$.

\section{Publications}

Tosso F., Hardy O. J., Doucet J.-L., Daïnou K., Kaymak E., Migliore J., 2018. Evolution in the Amphi-Atlantic tropical genus Guibourtia (Fabaceae, Detarioideae), combining NGS phylogeny and morphology. Molecular Phylogenetics and Evolution, 120: 83-93.

Tosso F., Doucet J.-L., Migliore J., Daïnou K., Kaymak E., Kameni F. S. M., Hardy O. J., 2017. Characterization of microsatellite markers in the African tropical tree species Guibourtia ehie (Fabaceae, Detarioideae). Applications in Plant Sciences, 5 (7).

Tosso F., Cherchye G., Hardy O. J., Daïnou K., Lognay G., Tagg N., Heuskin S., Haurez B., Souza A., Doucet J.-L., 2017. Characterization of animal communities involved in seed dispersal and predation of Guibourtia tessmannii (Harms) J. Léonard, a species newly listed on Appendix II of CITES. African Journal of Ecology.

Tosso F., Doucet J.-L., Kaymak E., Daïnou K., Duminil J., Hardy O. J., 2016. Microsatellite development for the genus Guibourtia (Fabaceae, Caesalpinioideae) reveals diploid and polyploid species. Applications in Plant Sciences, 4 (7).

Tosso F., Daïnou K., Hardy O. J., Sinsin B., Doucet J.-L., 2015. Le genre Guibourtia Benn., un taxon à haute valeur commerciale et sociétale (synthèse bibliographique). Biotechnologie, Agronomie, Société et Environnement, 19 (1) : 71-88.

Grade et diplôme : Docteur en Sciences agronomiques et ingénierie biologique. Université : Université de Liège, Gembloux Agro-Bio Tech, Belgique.

Date de soutenance : 9 février 2018.

Direction et encadrement : Professeur Jean-Louis DOUCET (Promoteur, Gembloux Agro-Bio Tech - Université de Liège, Belgique), Docteur Olivier J. HARDY (Copromoteur, Université Libre de Bruxelles, Belgique).

Composition du jury

Président : Professeur Philippe Lejeune (Président du Département BIOSE, Belgique).

Membres : Professeur Marc Aubinet (Gembloux Agro-Bio Tech - Université de Liège, Belgique), Docteur Adeline FAYolle (Gembloux Agro-Bio Tech - Université de Liège, Belgique), Docteur Alain HAMBuCKERS (Université de Liège, Belgique), Docteur Kasso DAïnou (Nature+, Belgique, Université Nationale d'Agriculture, Bénin), Professeur Thomas Couvreur (IRD, France), Professeur Achille AssogBADıO (Université d’Abomey-Calavi, Bénin).

Langue de rédaction : français et anglais.

\section{Accès au manuscrit :}

https://orbi.uliege.be/handle/2268/219892

Contact : Passage des déportés, 2, 5030 Gembloux,

Belgique.

dnftosso@uliege.be; tfelicien@yahoo.fr

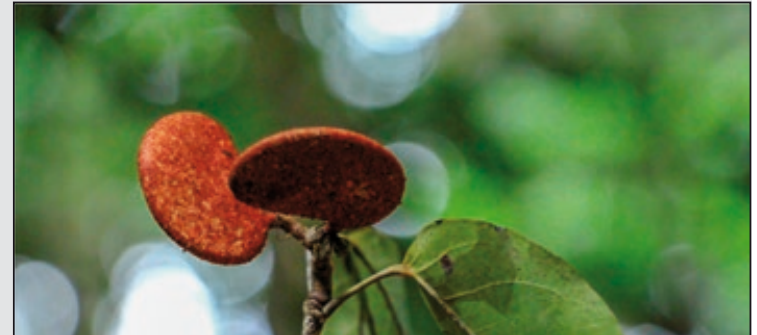

Photo 1.

Fruits de Guibourtia tessmannii contenant chacun une graine arillée dispersée par les animaux. Photo F. Tosso.

Photo 1.

Fruits of Guibourtia tessmannii, each containing an arillate seed dispersed by nimals. Photo F. Tosso.

\section{Foto 1.}

Frutas de Guibourtia tessmannii, cada una contiene una semilla arilada que será dispersada por los animales. Foto F. Tosso.

\section{Photo 2.}

Plantule de Guibourtia tessmannii (espèce inscrite sur l'annexe 2 de la CITES). Photo F. Tosso.

\section{Photo 2.}

Seedling of Guibourtia tessmannii (species listed in CITES Appendix II). Photo F. Tosso.

Foto 2.

Plántula de Guibourtia tessmannii (especie inscrita en el anexo 2 de la CITES). Foto F. Tosso 